\title{
Resposta a broncodilatador na espirometria: que parâmetros e valores são clinicamente relevantes em doenças obstrutivas?"
}

\author{
Roberto Rodrigues JR. ${ }^{1}$, Carlos Alberto de Castro Pereira ${ }^{2}$
}

\begin{abstract}
Respostas estatísticas dos parâmetros funcionais são largamente utilizadas após administração de broncodilatador $(\mathrm{Bd})$ nos laboratórios de função pulmonar em doenças com obstrução ao fluxo aéreo. Sua relevância clínica é discutivel. Objetivo: Determinar que parâmetros espirométricos refletem a melhora na tolerância ao exercício e na dispnéia em resposta a broncodilatador em doenças pulmonares obstrutivas. Métodos: 50 pacientes com DPOC e/ou asma $\left(\mathrm{VEF}_{1} / \mathrm{CVF}=41 \pm 11 \%\right)$ realizaram manobras de $\mathrm{CV}$ lenta e forçada, VVM e um teste de caminhada em corredor de seis minutos após treinamento, antes e após salbutamol, $400 \mathrm{mcg}$ fornecido por spray com espaçador. As respostas a broncodilatador foram expressas em valores absolutos, como incremento em relação ao

valor inicial e em relação aos valores previstos. Resposta após Bd foi considerada clinicamente significante quando a distância percorrida se elevou $30 \mathrm{~m}$ ou mais e/ou a dispnéia foi reduzida dois ou mais pontos com qualquer aumento na caminhada. Resultados: 32 pacientes foram considerados respondedores (R) e 18 não respondedores (NR). Como a distância caminhada em seis minutos se correlacionou com a idade ( $\mathrm{rs}=-0,38 ; \mathrm{p}<0,01$ ), análise dos dados espirométricos foi realizada incluindo a idade como covariada e expressa como $\mathrm{x} \pm \mathrm{EPE}$. $\mathrm{O}$ incremento do $\mathrm{VEF}_{1}$ não diferiu nos dois grupos: $\mathrm{R}=255 \pm 57 \mathrm{ml}, \mathrm{NR}=256 \pm 43 \mathrm{ml}$. Idem para a VVM: $\mathrm{R}=11 \pm 2 \mathrm{~L} / \mathrm{min}, \mathrm{NR}=10 \pm 2 \mathrm{~L} / \mathrm{min}$.

A melhor separação $(p<0,01)$ foi dada pela mudança da capacidade inspiratória (CI) seguida da mudança da capacidade vital lenta (CV): CI\% da inicial nos $\mathrm{R}=23 \pm 3 \%$ e os $\mathrm{NR}=3 \pm 4 \%$; CI absoluta: $\mathrm{R}=411 \pm 58 \mathrm{ml}, \mathrm{NR}=163 \pm 77 \mathrm{ml}$; Cl\% previsto: $\mathrm{R}=19 \pm 3 \%$ e $\mathrm{NR}=3 \pm 4 \%$. Para a CV os valores observados foram: $\mathrm{CV} \%$ do inicial $-\mathrm{R}=18 \pm 2 \%$ e $\mathrm{NR}=9 \pm 3 \%$; $\mathrm{CV}$ absoluta $-\mathrm{R}=448 \pm 52 \mathrm{ml}$ e NR

$=256 \pm 70 \mathrm{ml}$. Incrementos para a CI $>15 \%$ do inicial e $0,3 \mathrm{~L}$ e da $\mathrm{CV}>15 \%$ da inicial e 0,4L

separaram os respondedores com valor preditivo positivo (VPP) em torno de $90 \%$. Conclusão: A melhora do $\mathrm{VEF}_{1}$, CVF e VVM não prediz melhora na capacidade de exercício após Bd. Esta é melhor refletida por aumentos na CI e/ou CV acima de 15\% dos valores iniciais. (J Pneumol 2000;27(1):35-47)
\end{abstract}

\section{Spirometric response to bronchodilators: which parameters and values are clinically relevant in obstructive diseases?}

In lung function laboratories, statistical responses after bronchodilators (Bd) administration are widely used in patients with airflow limitation. However, their clinical relevance is debatable.

Objective: To determine which spirometric parameters best reflect improvement in both exercise tolerance and exertional dyspnea in response to bronchodilators in obstructive lung diseases.

Methods: Fifty patients with persistent asthma and/or COPD (FEV $/ F V C=41 \pm 11 \%)$ were submitted to slow and forced vital capacity testing, MVV and a six-minute walking test (6 MW) following practice, before and after a $400 \mu \mathrm{g}$ dose of salbutamol was given by MDI. Responses to

$B d$ were expressed in absolute values and considered as increments to the initial values and predicted values. Responses to $B d$ were considered clinically significant if distance was longer than $30 \mathrm{~m}$ in the $6 \mathrm{MW}$ test and/or if there was a 2-point decrease in the Borg scale in the walking distance. Results: 32 patients were responsive (R) to $B d$ and 18 were non-responsive (NR). As

* Trabalho realizado no Laboratório de Função Pulmonar do Hospital do Servidor Público Estadual, São Paulo, como parte de tese para obtenção do título de Mestre em Clínica Médica.

1. Pneumologista; Pós-Graduando em Clínica Médica.

2. Doutor em Pneumologia; Responsável pelo Setor de Função Pulmonar.
Endereço para correspondência - Av. Iraí, 393, conj. 34 - 04082 001 - São Paulo, SP. Tel./fax (11) 543-8070/240-8716; E-mail: pereirac@uol.com.br

Recebido para publicação em 7/4/00. Aprovado, após revisão, em 24/10/00. 
$\Delta M W$ correlated with age, $(r s=-0.38-p<0.01)$ an analysis on spirometric data, including age as covariant, was performed and expressed as $x \pm S E$. No difference in $F E V_{1}$ was observed between the groups $(R=255 \pm 57 \mathrm{ml} ; \mathrm{NR}=256 \pm 43 \mathrm{ml})$. The same occurred regarding $M V V(R=11 \pm 2 \mathrm{~L} / \mathrm{min}$; $N R=10 \pm 2 \mathrm{~L} / \mathrm{min}$.). The best separation $(p<0.01)$ was given by changes in inspiration capacity (IC) followed by changes in slow vital capacity (SVC): IC\% of initial $R=23 \pm 3 \%$ and NR $=3 \pm 4 \%$; absolute IC $=411 \pm 58 \mathrm{ml}$ and $\mathrm{NR}=163 \pm 77 \mathrm{ml}$; predicted IC\%: $R=19 \pm 3 \%$ and $\mathrm{NR}=3 \pm 4 \%$. For SVC, the values were: \% initial $-R=18 \pm 2 \%$ and $N R=9 \pm 3 \%$; absolute changes $-R=448$ $\pm 52 \mathrm{ml}$ and $\mathrm{NR}=256 \pm 70 \mathrm{ml}$. Increases in IC $>15 \%$ of the initial values and $0.3 \mathrm{~L}$ and

increases in SVS $>15 \%$ of the initial values and $0.4 \mathrm{~L}$ determined responsive patients with positive predicted values as approximately 90\%. Conclusion: Variations in FEV , FVC and MVV do not predict improvement in exercise capacity after $B d$. This is best reflected by $15 \%$ of increases in IC and/or in the SVC initial values.

Descritores - Pneumopatias obstrutivas. Espirometria. Valor preditivo dos testes. Broncodilatadores. Tolerância ao exercício. Dispnéia.

Key words - Obstructive lung diseases. Spirometry. Predictive value of tests. Bronchodilator agents. Exercise tolerance. Dyspnea.

\section{INTRODUÇÃO}

Testes de reversibilidade da obstrução ao fluxo aéreo são feitos de rotina nos laboratórios de função pulmonar. Diversas variações no $\mathrm{VEF}_{1}$ e na CVF, expressas de maneira absoluta ou relativa, têm sido propostas para caracterizar resposta ${ }^{(1,2)}$. Quando pacientes com obstrução ao fluxo aéreo são testados após placebo, ou sem medicação ativa, limites de variação das medidas funcionais são estabelecidos. Quando estes limites são excedidos, após broncodilatador, fica caracterizada resposta estatisticamente significante, isto é, além da variabilidade observada ao acaso. Incrementos de $0,2 \mathrm{~L}$ ou mais para o $\mathrm{VEF}_{1}$ e $0,35 \mathrm{~L}$ ou mais para a CVF são amplamente utilizados para caracterizar essa resposta ${ }^{(3,4)}$. Algumas dificuldades com essa abordagem são relevantes. Quando broncodilatadores são fornecidos a longo prazo para pacientes com DPOC, freqüentemente se observa melhora da dispnéia, sem que tenha havido mudança significativa do $\operatorname{VEF}_{1}(5,6)$. Quando pacientes com DPOC são repetidamente testados, mais de $2 / 3$ daqueles com testes iniciais negativos terão pelo menos um teste positivo ${ }^{(7)}$. Resposta estatística a $\mathrm{Bd}$ não é sinônimo de asma; DPOC e asma são melhor separados por incrementos de $\mathrm{VEF}_{1}$ abaixo ou acima de $10 \%$, respectivamente, do valor previsto ${ }^{(8,9)}$, porém muitos asmáticos, especialmente nos graus extremos de obstrução ao fluxo aéreo, respondem pouco ou nada ao Bd.

$\mathrm{O} \mathrm{VEF}_{1}$ é tido como o parâmetro funcional mais importante de resposta a $\mathrm{Bd}^{(10)}$, mas outros parâmetros devem ser considerados.

Após $\mathrm{Bd}$, a CPT usualmente permanece inalterada ${ }^{11-}$ 13), VR e CRF podem cair, o que resulta em elevação da capacidade vital lenta. Este achado pode ocorrer na au-
Siglas e abreviaturas utilizadas neste trabalho

ATS - American Thoracic Society

Bd - Broncodilatador

CI - Capacidade inspiratória

CPAP - Pressão positivo contínua nas vias aéreas

CPT - Capacidade pulmonar total

$\mathrm{CRF}$ - Capacidade residual funcional

$\mathrm{CV}$ - Capacidade vital

CVF - Capacidade vital forçada

CVFi - Capacidade vital forçada inspiratória

DCAM - Distância caminhada em metros

DP - Desvio padrão

EPE - Erro padrão de estimativa

FC - Freqüência cardíaca

$\mathrm{FIF}_{50}$ - Fluxo inspiratório forçado a $50 \%$ da CVFi

FIM - Fluxo inspiratório máximo

FR - Freqüência respiratória

HD - Hiperinsuflação dinâmica

IPAP - Pressão positiva inspiratória contínua nas vias aéreas

NR - Não respondedores

PEEP - Pressão endoexpiratória positiva

PEEP I - Pressão positiva expiratória intrínseca

PFE - Pico de fluxo expiratório

PFI - Pico de fluxo inspiratório

$\mathrm{R}$ - Respondedores

$\mathrm{SR}$ - Sistema respiratório

VAC - Volume de ar corrente

VE - Ventilação minuto

$\mathrm{VEF}_{1}$ - Volume expiratório forçado no primeiro segundo

VPP - Valor preditivo positivo

VR - Volume residual

VRE - Volume de reserva expiratória

VVM - Ventilação voluntária máxima

sência de mudança no $\mathrm{VEF}_{1}$ (resposta isolada de volume) $e$ se correlaciona com redução da dispnéia ${ }^{(14-18)}$.

$\mathrm{O}$ teste de caminhada de seis minutos ganhou larga aceitação como um teste simples para avaliar o desempenho de exercício em DPOC. Os resultados observados correlacionam-se melhor com os sintomas e a qualidade de vida do que os achados espirométricos e aqueles obtidos em exercício máximo ${ }^{(19,20)}$. Aumentos na distância percorrida acima de $30 \mathrm{~m}$, após diversas intervenções, exce- 
dem a variabilidade do teste e indicam resposta clinicamente relevante $e^{(21)}$.

Nos últimos 20 anos, diversos estudos demonstraram aumento na distância percorrida após broncodilatador, porém, de maneira inesperada, na maioria não se observou correlação entre este aumento e aqueles obtidos para $\mathrm{O} \mathrm{VEF}_{1}$ e a CVF ${ }^{(22-24)}$. Em vários pacientes a distância caminhada não se alterou, mas a dispnéia foi reduzida(23,25).

Pacientes com asma e DPOC referem que a dispnéia é maior durante a inspiração(26). Durante o exercício a limitação ao fluxo aéreo resulta em respiração em volumes pulmonares elevados (hiperinsuflação dinâmica), o que aumenta o trabalho elástico. Pacientes com DPOC hiperinsuflados têm mais dispnéia e maior limitação aos esforços, quando comparados com os demais com grau semelhante de obstrução ao fluxo aéreo(27). Após broncodilatador, pode ocorrer elevação da capacidade inspiratória, o que reflete a redução da hiperinsuflação dinâmica, sem que haja mudança significativa do $\operatorname{VEF}_{1}{ }_{1}^{(13,27,28)}$. O Bd, ao diminuir a hiperinsuflação dinâmica durante o esforço, reduz a dispnéia e permite melhor desempenho em exercícios realizados com cargas fixas ${ }^{(29,30)}$. A hiperinsuflação dinâmica durante o exercício pode ser medida pela capacidade inspiratória (que, por definição, se reduz) durante o teste. A variação da capacidade inspiratória após $\mathrm{Bd}$, medida em repouso, poderia, como a capacidade inspiratória medida no exercício, relacionar-se com melhor desempenho no esforço.

$\mathrm{Na}$ alça fluxo-volume, os fluxos inspiratórios são determinados pela resistência das vias aéreas e pela potência dos músculos respiratórios. Aumentos dos fluxos inspiratórios poderiam refletir a desinsuflação pulmonar após $\mathrm{Bd}$, com conseqüente melhora na potência dos músculos inspiratórios, ou broncodilatação não observada na alça expiratória, por efeito de compressão dinâmica das vias aéreas.

Em DPOC a VVM tem alta correlação com o $V_{E F}$. Em pacientes com doenças obstrutivas, na manobra da VVM, o alçaponamento de ar é facilmente aparente como aumento progressivo na posição endoexpiratória, à semelhança do que ocorre em exercício. O aumento da VVM após Bd poderia demonstrar, além da broncodilatação, a redução da hiperinsuflação.

Os objetivos do presente estudo foram:

1) Estabelecer que parâmetros funcionais indicam resposta clinicamente significante, caracterizada por teste de caminhada após Bd.

2) Determinar, dentre os parâmetros eventualmente selecionados, os pontos de corte para caracterizar os respondedores.

3) Avaliar o valor preditivo dos testes estatísticos mais comuns propostos na literatura para caracterizar resposta no exercício após Bd.

\section{MATERIAL E MÉTODOS}

Cinqüenta pacientes ( 42 homens e oito mulheres, com média de idade \pm DP de $62 \pm 12$ anos (variando de 36 a 84), foram avaliados numa condição estável. Trinta e sete tinham diagnóstico de DPOC, três de asma persistente $e$ dez de DPOC + asma(31).

Todos tinham obstrução moderada ou acentuada ao fluxo aéreo ${ }^{(32)}$ de acordo com os valores previstos para a população brasileira(33) $-\mathrm{VEF}_{1}: 36 \pm 14 \%$ ou $1,06 \pm 0,42 \mathrm{~L}$ e relação $\mathrm{VEF}_{1} / \mathrm{CVF}=42 \pm 11 \%$. Outra doenças respiratórias e cardíacas estavam ausentes. Todos estavam livres de afecções músculo-esqueléticas, com prejuízo para a deambulação. Broncodilatadores de curta duração foram suspensos oito horas antes do estudo e, de longa duração, 24 horas antes.

Quarenta e nove pacientes eram ou foram fumantes de $49 \pm 28$ anos-maço.

Espirometria foi realizada utilizando-se um espirômetro Collins DSII, com calibração verificada diariamente. Manobras de CV lenta, CVF expiratória e inspiratória $e$ VVM foram obtidas nesta ordem e aceitas segundo os critérios propostos pela $\mathrm{ATS}^{(34)}$, exceto que $\mathrm{VEF}_{1}$ selecionado, antes e após Bd, foi o maior das três curvas aceitáveis com diferença no pico de fluxo expiratório abaixo de 0,5L/ $\mathrm{s}^{(32)}$. A pausa após a inspiração máxima até a CPT, precedendo a expiração máxima, foi menor que três segundos.

$\mathrm{Na}$ CV lenta, os dois melhores valores não deveriam diferir mais de 0,2L; para a capacidade inspiratória, o valor selecionado foi o da curva com maior CV. Para aceitação da CV lenta e da CI, nível endoexpiratório consistente e volumes expiratórios e inspiratórios completos deveriam ser obtidos, confirmados pelos platôs de volume alcançados assintoticamente. Valores previstos para CI foram baseados na diferença entre $\mathrm{CPT}$ e $\mathrm{CRF}^{(35)}$. Na análise das curvas de fluxo-volume inspiratórias máximas, as de maiores valores foram selecionadas. Valores normais foram baseados em Bass(36).

Para aceitação, as duas melhores manobras para a CVFi não deveriam diferir mais de $10 \%$ da $\mathrm{CVF}$ e o $\mathrm{FIF}_{50}$ e o PFI não deveriam diferir mais de $20 \%$.

A manobra da VVM foi realizada em triplicata. Valores normais foram baseados em Neder et al. (37). Outras manobras de VVM foram a seguir obtidas em 38 pacientes no modo de obtenção da CV lenta. Após estabilização da linha de base, manobras de CI foram obtidas (CI "dinâmicas") para simular a Cl em exercício. O maior valor obtido foi selecionado para análise.

A seguir, foi realizado teste de caminhada em corredor, por seis minutos. Este foi precedido por práticas de caminhada e leitura da escala de Borg ${ }^{(6)}$.

Um dos autores realizou os testes de caminhada acompanhando os pacientes durante todo o trajeto, sustentan- 
do um oxímetro conectado ao doente; estímulos verbais eram dados em intervalos. O ritmo da caminhada era determinado pelo paciente. Ao final de seis minutos, foram anotados a distância percorrida, FC, FR, e a dispnéia foi medida por escala de Borg. Após repouso foi administrado salbutamol, $400 \mathrm{mcg}$, através de um nebulímetro pressurizado por câmara de expansão e todos os procedimentos foram repetidos na mesma ordem.

Vinte e nove pacientes caminharam $\geq 30 \mathrm{~m}$ após o $\mathrm{Bd}^{(21)}$. Treze pacientes tiveram redução da dispnéia considerada significativa (redução de dois ou mais pontos na escala de Borg para dispnéia); destes, nove caminharam mais de 30 metros após o Bd; dos quatro restantes, dois tinham queda de três pontos na escala de Borg, com variação na DCAM de zero e +25 metros e um teve queda de dois pontos na dispnéia com incremento da caminhada de $27 \mathrm{~m}$ após Bd. Estes foram incluídos no grupo dos respondedores, perfazendo 32 pacientes. O quarto paciente teve redução de dois pontos na escala de Borg, porém a DCAM após Bd foi de cinco metros a menos. Este e os outros 17 foram considerados não respondedores.

Os 13 pacientes no presente estudo que tiveram redução de dois ou mais pontos na escala de Borg para a dispnéia percorreram, após o $\mathrm{Bd}, 81 \pm 14$ metros a mais $\left(\mathrm{IC}_{95}=53\right.$ a $\left.109 \mathrm{~m}\right)$, comparados com $35 \pm 8 \mathrm{~m}\left(\mathrm{IC}_{95}=18\right.$ a $51 \mathrm{~m})$ nos 37 restantes. Doentes com redução de um ponto na escala da dispnéia e pacientes sem redução da dispnéia aumentaram a distância caminhada de maneira semelhante (35 metros).

Caracterizados os grupos respondedor e não respondedor no teste de caminhada, os incrementos nos parâmetros funcionais, expressos como variação absoluta,

TABELA 1

Valores para a espirometria e $\mathrm{SatO}_{2}$, antes e após broncodilatador, obtidos em 50 portadores de obstrução ao fluxo aéreo

\begin{tabular}{|c|c|c|c|c|}
\hline Variável & $\begin{array}{l}\text { Pré-Bd } \\
x \pm D P\end{array}$ & $\begin{array}{l}\text { Pós-Bd } \\
x \pm D P\end{array}$ & $\mathbf{Z}$ & $\mathbf{p}$ \\
\hline $\mathrm{Cl}(\mathrm{L})$ & $2,05 \pm 0,55$ & $2,34 \pm 0,62$ & 4,70 & $<0,001$ \\
\hline VRE (L) & $0,97 \pm 0,41$ & $1,09 \pm 0,44$ & 2,34 & 0,02 \\
\hline $\mathrm{CV}(\mathrm{L})$ & $3,02 \pm 0,74$ & $3,41 \pm 0,75$ & 5,98 & $<0,001$ \\
\hline CVF (L) & $2,56 \pm 0,68$ & $3,09 \pm 0,78$ & 6,03 & $<0,001$ \\
\hline $\operatorname{VEF}_{1}(\mathrm{~L})$ & $1,06 \pm 0,42$ & $1,32 \pm 0,54$ & 5,98 & $<0,001$ \\
\hline VVM (L) & $43 \pm 18$ & $54 \pm 21$ & 5,65 & $<0,001$ \\
\hline PFE (L/s) & $3,37 \pm 1,36$ & $3,70 \pm 1,41$ & 2,84 & $<0,001$ \\
\hline $\mathrm{FEF}_{50}(\mathrm{~L} / \mathrm{s})$ & $0,40 \pm 0,25$ & $0,53 \pm 0,38$ & 4,53 & $<0,001$ \\
\hline $\mathrm{FEF}_{25-75 \%}(\mathrm{~L} / \mathrm{s})$ & $0,34 \pm 0,15$ & $0,44 \pm 0,25$ & 5,50 & $<0,001$ \\
\hline $\mathrm{FEF}_{75-85}(\mathrm{~L} / \mathrm{s})$ & $0,11 \pm 0,04$ & $0,14 \pm 0,07$ & 4,29 & $<0,001$ \\
\hline $\mathrm{PFI}(\mathrm{L} / \mathrm{s}, \mathrm{n}=41)$ & $3,60 \pm 1,29$ & $3,96 \pm 1,38$ & 2,10 & 0,04 \\
\hline $\mathrm{FIF}_{50}(\mathrm{~L} / \mathrm{s}, \mathrm{n}=45)$ & $3,45 \pm 1,30$ & $3,76 \pm 1,34$ & 1,47 & 0,14 \\
\hline $\operatorname{Texp}(\mathrm{s})$ & $11,5 \pm 1,9$ & $11,7 \pm 2,2$ & 1,00 & 0,31 \\
\hline $\mathrm{SatO}_{2}$, rep (\%) & $94,0 \pm 2,9$ & $94,1 \pm 2,5$ & 0,35 & 0,73 \\
\hline
\end{tabular}

como incremento percentual em relação aos valores iniciais e em relação aos valores previstos, foram comparados nos dois grupos, e calculado o intervalo de confiança de $95 \%$ para as médias. Como houve correlação negativa significante entre a variação na distância percorrida $e$ a idade ( $\mathrm{rs}=-0,38 ; \mathrm{p}=0,008$ ), as diferenças entre os grupos foram comparadas tendo a idade como covariada.

Os dados gerais foram expressos como $\mathrm{x} \pm \mathrm{DP} e \mathrm{x} \pm$ EPE. De acordo com a distribuição dos dados, comparações entre dois grupos foram feitas por teste de $t$ de Student ou teste de Mann-Whitney e correlações foram calculadas pelos coeficientes de Pearson e de Spearman. Os dados foram analisados pelo pacote estatístico Statgraphics $5.1 \mathrm{em}$ um computador pessoal. Valores de p foram considerados significativos se $\alpha \leq 0,05$.

\section{REsUlTADOS}

Os valores obtidos para os dados da curva expiratória forçada, capacidade vital lenta e VVM se elevaram significativamente após $\mathrm{Bd}$. Os fluxos inspiratórios em média também se elevaram, mas, pela larga variabilidade, as mudanças não foram significativas. $\mathrm{A} \mathrm{SatO}_{2}$ de repouso não se alterou (Tabela 1).

O tempo expiratório da manobra da CVF permaneceu em média inalterado. Houve fraca, porém significativa, correlação direta entre a variação da CVF e a variação do tempo expiratório $(r=0,32 ; p=0,02)$. A variação do tempo expiratório não se correlacionou com a variação da distância caminhada nem com a variação da dispnéia.

A resposta a $\mathrm{Bd}$ foi significativamente maior, na espirometria, nos 13 pacientes com asma isolada ou associada a DPOC em comparação com os 37 portadores de DPOC isolada: variação absoluta do $\mathrm{VEF}_{1}=0,34 \pm 0,21 \mathrm{~L}$ vs. $0,19 \pm 0,21 \mathrm{~L}(\mathrm{p}=0,001)$, respectivamente.

Os achados observados no teste de caminhada, antes e após Bd, são mostrados na Tabela 2 .

A distância percorrida elevou-se de maneira significativa, em média, $42 \mathrm{~m}$ após o Bd. A dispnéia reduziu-se em

TABELA 2

Valores observados ao final do teste de caminhada de 6 minutos, antes e após broncodilatador, em 50 portadores de obstrução ao fluxo aéreo

\begin{tabular}{lcccc}
\hline \multicolumn{1}{c}{ Variável } & $\begin{array}{c}\text { Pré-Bd } \\
\mathbf{x} \pm \mathbf{D P}\end{array}$ & $\begin{array}{c}\text { Pós-Bd } \\
\mathbf{x} \pm \mathbf{D P}\end{array}$ & $\mathbf{Z}$ & $\mathbf{p}$ \\
\hline $\mathrm{fr}(\mathrm{rpm})$ & $29 \pm 5$ & $28 \pm 5$ & 2,34 & 0,02 \\
$\mathrm{fC}(\mathrm{bpm})$ & $115 \pm 14$ & $114 \pm 14$ & 1,38 & 0,17 \\
$\mathrm{SatO}_{2}(\%)$ & $88,9 \pm 5,5$ & $89,7 \pm 5,3$ & 1,96 & 0,05 \\
DCAM $(\mathrm{m})_{\text {Dispnéia, Borg }}$ & $409 \pm 166$ & $451 \pm 166$ & 5,04 & $<0,001$ \\
& $4,4 \pm 2,6$ & $3,3 \pm 2,0$ & 4,45 & $<0,001$ \\
\hline
\end{tabular}


média um ponto, e a saturação de $\mathrm{O}_{2}$ se elevou, em média, $1 \%$. Exceto para a freqüência cardíaca, estas mudanças foram estatisticamente significantes; embora de pequena magnitude, as tendências intra-individuais foram consistentes para a saturação de $\mathrm{O}_{2}$ e dispnéia. Pelo critério de resposta ao $\mathrm{Bd}$ no teste de caminhada (DCAM $\geq$ $30 \mathrm{~m}$ ou DCAM $>0 \mathrm{~m}$ com redução da dispnéia $\geq$ dois pontos), 32 pacientes foram considerados respondedores $e$ 18 não respondedores.

A idade foi semelhante nos dois grupos $(\mathrm{R}=61 \pm 11$ vs. NR $65 \pm 11$ anos, $\mathrm{p}=0,22$ ), porém o $\mathrm{VEF}_{1}$ e a $\mathrm{CV}$ eram menores nos respondedores: $\mathrm{VEF}_{1}-\mathrm{R}=33 \pm 13 \%$, $\mathrm{NR}=43 \pm 13 \%, \mathrm{p}=0,02 ; \mathrm{CV}-\mathrm{R}=74 \pm 14 \%$, NR $=91$ $\pm 14 \%, p=0,0002$. A CVF não diferiu entre os dois grupos $-\mathrm{R}=63 \pm 18 \%, \mathrm{NR}=64 \pm 19 \%(\mathrm{p}=0,77)$. A saturação de $\mathrm{O}_{2}$ pré-Bd, ao final da caminhada, foi nos respondedores de $89 \pm 5 \%$ comparada com $91 \pm 5 \%$ nos não respondedores $(p=0,12)$. A saturação de repouso também não diferiu entre os dois grupos.

As variações observadas após $\mathrm{Bd}$ nos diversos parâmetros funcionais, nos grupos $\mathrm{R} e \mathrm{NR}$, são mostradas na Tabela 3 .

As variações para o $\mathrm{VEF}_{1}$, fluxos expiratórios e inspiratórios máximos, VVM e $\mathrm{SatO}_{2}$ ex, não diferiram entre os dois grupos. A dispnéia diminuiu de maneira significativa no grupo respondedor em comparação com o grupo não respondedor.

Fica evidente que a melhor separação foi dada pelas mudanças da CI, seguida da CV. Com base no intervalo de confiança de $95 \%$ da média, pontos de corte foram selecionados e a sensibilidade, especificidade, e valores preditivos positivo e negativo foram calculados (Tabela 4).

TABELA 3

Mudanças para os dados de espirometria, $\mathrm{SatO}_{2}$ e dispnéia após broncodilatador observadas em pacientes com obstrução ao fluxo aéreo, separados por resposta no teste de caminhada (DCAM $\geq 30 \mathrm{~m}$ e/ou DCAM $>0 \mathrm{~m}$ e redução dispnéia $\geq 2$ pontos) ou não resposta

\begin{tabular}{|c|c|c|c|c|c|}
\hline \multirow[t]{2}{*}{ Mudança } & \multicolumn{2}{|c|}{ Respondedores $(\mathrm{n}=32)$} & \multicolumn{2}{|c|}{ Não respondedores $(n=18)$} & \multirow[t]{2}{*}{$\mathbf{p}$} \\
\hline & $x \pm E P E$ & IC 95 & $x \pm E P E$ & IC 95 & \\
\hline $\mathrm{Cl}$ abs $(\mathrm{ml})$ & $410 \pm 56$ & 297 a 524 & $86 \pm 76$ & -66 a 238 & 0,0013 \\
\hline$\%$ inic. & $23 \pm 3$ & 17 a 30 & $4 \pm 4$ & -5 a 12 & 0,0005 \\
\hline \% prev. & $19 \pm 3$ & 13 a 24 & $4 \pm 4$ & -3 a 11 & 0,0027 \\
\hline CV abs (ml) & $488 \pm 53$ & 383 a 594 & $242 \pm 70$ & 100 a 384 & 0,0077 \\
\hline$\%$ inic. & $18 \pm 2$ & 14 a 22 & $8 \pm 3$ & 3 a 14 & 0,0096 \\
\hline \% prev. & $13 \pm 1$ & 10 a 16 & $7 \pm 2$ & 3 a 11 & 0,011 \\
\hline CVF abs (ml) & $582 \pm 66$ & 448 a 715 & $438 \pm 89$ & 259 a 617 & 0,20 \\
\hline$\%$ inic. & $25 \pm 3$ & 20 a 31 & $16 \pm 4$ & 9 a 24 & 0,07 \\
\hline \% prev. & $15 \pm 2$ & 12 a 19 & $12 \pm 2$ & 8 a 17 & 0,30 \\
\hline $\mathrm{VEF}_{1}$ abs (ml) & $256 \pm 43$ & 170 a 342 & $255 \pm 57$ & 140 a 370 & 0,99 \\
\hline$\%$ inic. & $27 \pm 4$ & 20 a 35 & $23 \pm 15$ & 13 a 33 & 0,52 \\
\hline \% prev. & $8 \pm 1$ & 6 a 11 & $9 \pm 2$ & 6 a 12 & 0,79 \\
\hline $\mathrm{FIF}_{50}$ abs (L/s) & $0,46 \pm 0,19$ & 67 a 854 & $0,00 \pm 0,27$ & $-0,55$ a 0,54 & 0,17 \\
\hline$\%$ inic. & $17 \pm 7$ & 4 a 31 & $7 \pm 9$ & -12 a 25 & 0,37 \\
\hline \% prev. & $11 \pm 4$ & 2 a 20 & $-1 \pm 6$ & -13 a 12 & 0,14 \\
\hline \multicolumn{6}{|l|}{$\mathrm{FEF}_{50}$ iso-basal } \\
\hline Abs. & $343 \pm 80$ & 183 a 503 & $382 \pm 107$ & 168 a 596 & 0,78 \\
\hline$\%$ inic. & $88 \pm 17$ & 54 a 122 & $106 \pm 23$ & 60 a 152 & 0,54 \\
\hline \% prev. & $9 \pm 2$ & 5 a 13 & $11 \pm 3$ & 5 a 16 & 0,65 \\
\hline $\mathrm{FEF}_{75-85}$ Abs. & $20 \pm 8$ & 4 a 37 & $40 \pm 11$ & 17 a 62 & 0,18 \\
\hline$\%$ inic. & $18 \pm 7$ & 4 a 32 & $36 \pm 9$ & 17 a 55 & 0,14 \\
\hline VVM abs (ml) & $11 \pm 2$ & 7 a 15 & $10 \pm 3$ & 5 a 15 & 0,64 \\
\hline$\%$ inic. & $31 \pm 5$ & 21 a 41 & $22 \pm 6$ & 9 a 35 & 0,29 \\
\hline \% prev. & $8 \pm 2$ & 5 a 11 & $8 \pm 2$ & 4 a 12 & 0,96 \\
\hline PFE (L/s) & $0,35 \pm 0,14$ & 0,06 a 0,64 & $0,30 \pm 0,19$ & $-0,08$ a 0,69 & 0,85 \\
\hline $\mathrm{FEF}_{50}(\mathrm{~L} / \mathrm{s})$ & $0,11 \pm 0,04$ & 0,03 a 0,19 & $0,15 \pm 0,05$ & 0,05 a 0,26 & 0,50 \\
\hline $\mathrm{FEF}_{25-75}(\mathrm{~L} / \mathrm{s})$ & $0,09 \pm 0,02$ & 0,04 a 0,14 & $0,11 \pm 0,03$ & 0,05 a 0,18 & 0,55 \\
\hline $\mathrm{PFI}(\mathrm{L} / \mathrm{s})$ & $0,52 \pm 0,19 *$ & 0,13 a 0,91 & $0,07 \pm 0,27^{* *}$ & $-0,47$ a 0,61 & 0,18 \\
\hline $\mathrm{SatO}_{2} \mathrm{ex}(\%)$ & $1,1 \pm 0,5$ & 0,2 a 2,0 & $0,2 \pm 0,6$ & $-1,0$ a 1,5 & 0,26 \\
\hline Dispnéia, Borg & $-1,4 \pm 0,26$ & $-1,9$ a $-0,9$ & $-5,0 \pm 0,3$ & $-1,2$ a 0,2 & 0,05 \\
\hline
\end{tabular}

$* \mathrm{n}=27 ; * * \mathrm{n}=14$ 
TABELA 4

Sensibilidade e especificidade das mudanças após Bd de alguns parâmetros funcionais como indicadores de melhor desempenho no teste de caminhada de 6 minutos ${ }^{(*)}$, em 50 portadores de obstrução ao fluxo aéreo

\begin{tabular}{lccrr}
\hline \multicolumn{1}{c}{ Variável } & $\begin{array}{c}\text { Sensibilidade } \\
(\mathbf{\%})\end{array}$ & $\begin{array}{c}\text { Especificidade } \\
(\mathbf{\%})\end{array}$ & VPP & VPN \\
\hline $\mathrm{Cl} \geq 0,3 \mathrm{~L}$ & 62 & 83 & 87 & 5 \\
$\mathrm{Cl} \geq 15 \%$ inicial & 66 & 89 & 91 & 59 \\
$\mathrm{Cl}>10 \%$ previsto & 69 & 83 & 81 & 57 \\
$\mathrm{CV} \geq 0,40 \mathrm{~L}$ & 56 & 72 & 78 & 48 \\
$\mathrm{CV} \geq 15 \%$ inicial & 56 & 72 & 78 & 48 \\
$\mathrm{CV}>10 \%$ previsto & 56 & 67 & 75 & 75 \\
$\mathrm{CV}+\mathrm{Cl} \geq 25 \%$ inicial & 69 & 89 & 92 & 62 \\
$\mathrm{Cl}$ e/ou CV $\geq 15 \%$ inicial & 78 & 72 & 83 & 65 \\
\hline
\end{tabular}

* Aumento de $30 \mathrm{~m}$ ou mais ou queda dispnéia $\geq 2$ pontos (Borg) com qualquer aumento na DCAM

$\mathrm{VPP}=$ valor preditivo positivo

$\mathrm{VPN}=$ valor preditivo negativo

Os valores preditivos positivos para a $\mathrm{CI}$ situaram-se em torno de $90 \%$ e, para a $\mathrm{CV}$, em torno de $80 \%$. Os valores preditivos negativos situaram-se em patamares abaixo de $60 \%$.

Mudanças de $15 \%$ ou mais em relação ao valor inicial para a $\mathrm{Cl}$ e $\mathrm{CV}$ classificaram os pacientes com acurácia de $76 \%$. Quando as respostas individuais foram analisadas por estes pontos de corte, 14 pacientes responderam com aumento da $\mathrm{Cl}$ e $\mathrm{CV} \geq 15 \%$ do valor inicial, nove apenas pela $\mathrm{CI} \geq 15 \%$ e oito apenas pela $\mathrm{CV} \geq 15 \%$; em 19 não houve resposta por estes critérios. A distância caminhada após seis minutos nos oito respondedores isolados pela CV elevou-se $69 \pm 16 \mathrm{~m}$ comparada com $43 \pm 15$ nos nove com resposta isolada pela $\mathrm{CI}(\mathrm{t}=3,44 ; \mathrm{p}<0,01)$. Quando ambos os critérios de resposta foram preenchidos, a distância percorrida elevou-se $71 \pm 12 \mathrm{~m}$ e, quando nenhum critério foi alcançado, a distância variou $22 \pm$ $10 \mathrm{~m}$. Quando a variação da dispnéia após a caminhada foi correlacionada com a variação dos parâmetros funcionais, correlações significativas foram observadas entre variação da $\mathrm{CV}$ (\% inicial, $r \mathrm{~s}=0,38, \mathrm{p}=0,007)$, mas não com a $\mathrm{CI}$ ( $\mathrm{rs}=0,17, \mathrm{p}=0,24)$.

A variação da $\mathrm{CI}$ após $\mathrm{Bd}$, expressa como percentagem do valor inicial, correlacionou-se com o valor absoluto inicial da própria $\mathrm{CI}(\mathrm{r}=-0,34 ; \mathrm{p}=0,002)$, o que não ocorreu quando expressa como variação absoluta $(r=$ $-0,10 ; p=0,48)$ ou em relação ao valor previsto $(r=$ $-0,19 ; p=0,18)$. A variação da $C V$ quando expressa em valores absolutos não se correlacionou com os valores basais $(r=-0,18 ; p=0,20)$, porém houve correlação significativa quando expressa como percentagem do valor inicial $(r=-0,43 ; p=0,002)$ ou percentagem do previsto $(r=-0,28 ; p=0,049)$.

À semelhança do $\mathrm{VEF}_{1}$, a medida da VVM não separou os respondedores.

Na manobra da VVM, houve elevação significativa da linha de base, expressa pela redução da $\mathrm{CI}$, antes do $\mathrm{Bd}$ (de 2,00 \pm 0,54 na manobra de CV lenta para 1,16 \pm $0,45 \mathrm{~L}$ na manobra da VVM, $\mathrm{p}<0,001)$. Após Bd a CI da manobra da CV lenta se elevou para 2,28 $\pm 0,60$ e a CI da manobra da VVM para 1,42 $\pm 0,57 \mathrm{~L}$. Estes dados são esquematizados na Figura 1.

O aumento da CI na manobra da VVM (CI "dinâmica") após Bd foi maior nos respondedores $(\mathrm{n}=24, \mathrm{x}=346 \pm$ $60 \mathrm{ml}$ ) em comparação com os não respondedores $(\mathrm{n}=$ $14, \mathrm{x}=128 \pm 79, \mathrm{p}<0,01)$.

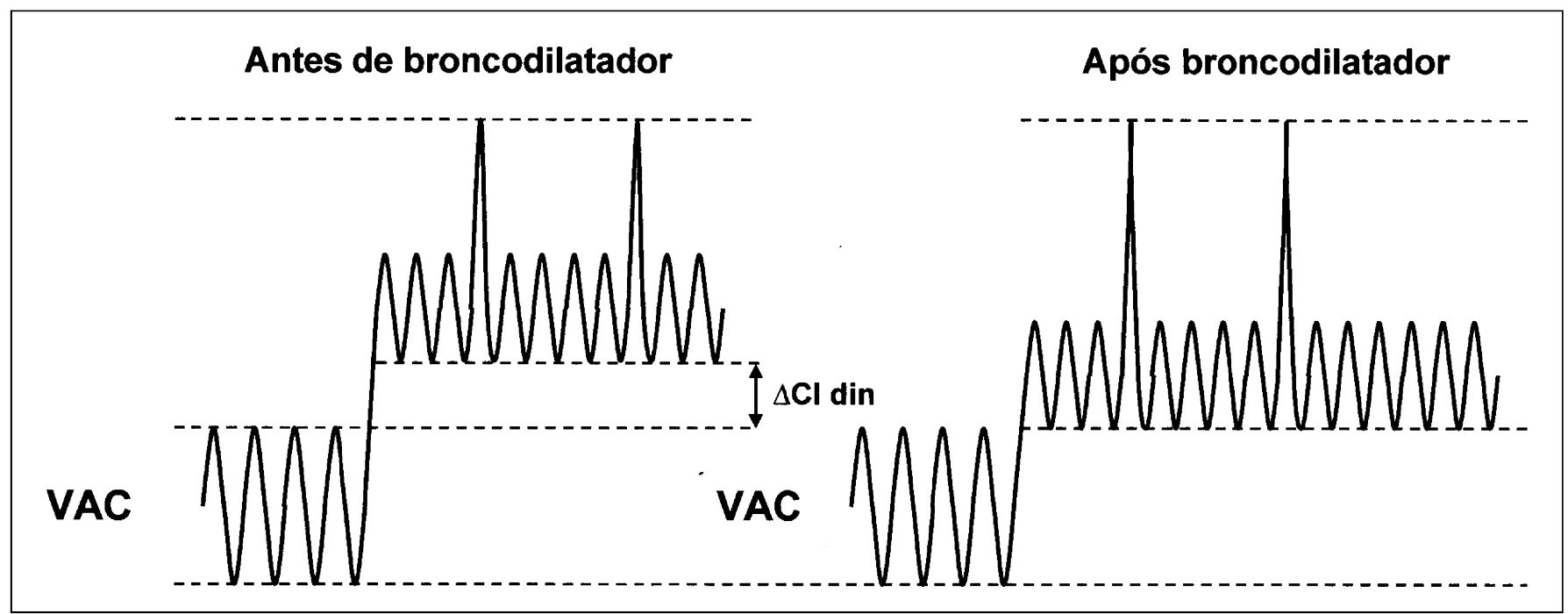

Figura 1 - Aumento da "capacidade inspiratória dinâmica" após Bd, na manobra de VVM por efeito de redução da hiperinsuflação. No exercício os achados são semelhantes. 
TABELA 5

Sensibilidade e especificidade das mudanças do $\mathrm{VEF}_{1}$ após broncodilatador propostas na literatura como indicadores de melhor desempenho no teste de caminhada de 6 minutos ${ }^{(*)}$ em 50 portadores de obstrução ao fluxo aéreo

Critério (referência) Sensibilidade Especificidade VPP VPN (\%) (\%)

\begin{tabular}{|c|c|c|c|}
\hline $\mathrm{VEF}_{1} \geq 0,2 \mathrm{~L}$ e $12 \%{ }^{(38)}$ & 47 & 39 & 58 \\
\hline $\mathrm{VEF}_{1}>7 \%$ prev. e $0,2 \mathrm{~L}^{(32)}$ & 47 & 39 & 58 \\
\hline $\operatorname{VEF}_{1}>10 \%$ prev $^{(9)}$ & 34 & 56 & 58 \\
\hline $\mathrm{VEF}_{1}>0,2 \mathrm{~L}^{(39)}$ & 47 & 39 & 58 \\
\hline
\end{tabular}

* DCAM $\geq 30 \mathrm{~m}$ e/ou DCAM $>0 \mathrm{~m}$ e redução dispnéia $\geq 2$ pontos

Embora os fluxos inspiratórios não se tenham elevado de maneira significativa, houve correlação de sua variação com as variações da CVF $(r=0,65 ; p \leq 0,001), C V(r$ $=0,60 ; p \leq 0,001), \mathrm{CI}(\mathrm{r}=0,50 ; \mathrm{p}=0,001)$ e $\mathrm{VEF}_{1}(\mathrm{r}=$ $0,45 ; p=0,003)$, porém não com o PFE $(r=0,19 ; p=$ $0,22)$ e o $\mathrm{FEF}_{50}(\mathrm{r}=0,17 ; \mathrm{p}=0,29)$. Por análise multivariada, a CVF e a CI permaneceram significantes $\left(\mathrm{r}^{2}=0,48\right)$.

Diversos critérios estatísticos foram propostos por várias sociedades para caracterizar resposta significativa ao $\mathrm{Bd}$ pelo $\mathrm{VEF}_{1}$. A sensibilidade e a especificidade desses critérios em relação ao proposto no presente estudo, pelo teste de caminhada, são mostradas na Tabela 5.

Vinte e seis pacientes responderam ao Bd pelo critério proposto pela ATS (VEF $>$ $>0,2 \mathrm{~L}$ e $12 \%$ do inicial). Destes, 15 tiveram melhora no desempenho do exercício e 11, não (VPP $=58 \%$ ). Os outros critérios propostos resultaram igualmente em valores preditivos positivos insuficientes e valores preditivos negativos muito baixos, isto é, muitos pacientes sem resposta por estes critérios terão melhora clínica.

\section{DISCUSSÃO}

\section{1) Bds e teste de caminhada}

Quando Bds são fornecidos para pacientes com obstrução ao fluxo aéreo, observa-se em geral aumento na distância percorrida no teste de caminhada; em alguns estudos, observou-se redução na dispnéia, com distância percorrida inalterada(23,25). Quando a variação na distância caminhada pós-Bd foi comparada com as mudanças observadas na espirometria, correlações em geral não foram encontradas, o que levou alguns autores a considerar que o alívio da obstrução não seria a única explicação para o melhor desempenho ${ }^{(22-24)}$.

Berger e Smith, em 1988(22), observaram que a DCAM aumentou $82 \mathrm{~m}$ após Bd a despeito da melhora mínima no $\mathrm{VEF}_{1}$ e na CVF. Reviram os estudos anteriores e notaram que em sete havia relato de melhora dos sintomas ou no desempenho de exercício, sem melhora funcional. Con- sideraram inexplicados esses achados. Hay et al., em $1992^{(23)}$, classificaram 32 doentes com DPOC como respondedores ou não ao $\mathrm{Bd}$, por resposta do $\mathrm{VEF}_{1}$ e da CVF, por incremento funcional excedendo a variabilidade intra-individual (140ml para o $\mathrm{VEF}_{1}$ e $390 \mathrm{ml}$ para a CVF). Não houve correlação entre a resposta funcional e a melhora no teste de caminhada. Observaram ainda que, após o $\mathrm{Bd}$, alguns doentes percorriam a mesma distância, com dispnéia menor. Houve correlação significativa entre mudança na dispnéia em repouso e variação na distância caminhada $(r=-0,44 ; p<0,01)$. Sugeriram que a diminuição do PEEPI poderia explicar os achados.

Wolkove et al. ${ }^{(40)}$ notaram que, após $\mathrm{Bd}$, dez pacientes referiam redução de três ou mais pontos na escala de Borg em repouso e, destes, seis tinham aumento do $\mathrm{VEF}_{1}$ menor que $0,1 \mathrm{~L}$.

Guyatt et al. ${ }^{(21)}$, após extensa experiência com teste de caminhada, sugeriram que incrementos de $30 \mathrm{~m}$ ou mais expressam resposta clinicamente relevante.

No presente estudo, a distância percorrida após Bd se elevou, em média, $42 \mathrm{~m}$ e a dispnéia diminuiu um ou mais pontos em 27 pacientes (54\%). A distância percorrida antes do Bd correlacionou-se com a dispnéia ao final do teste ( $\mathrm{rs}=0,58 ; \mathrm{p}<0,001)$ e com o $\mathrm{VEF}_{1}$ ( $\mathrm{rs}=0,50 ; \mathrm{p}<$ 0,001 ), porém, quando as mudanças após o Bd foram consideradas, não houve correlação significativa com a variação do $\operatorname{VEF}_{1}$ ( $\left.r s=0,17 ; p=0,75\right)$. A mudança na distância caminhada correlacionou-se com a variação da dispnéia ( $r s=-0,40 ; p=0,004)$. Como pode haver significativa redução da dispnéia sem que a distância percorrida se eleve acentuadamente, redução de dois ou mais pontos na escala de Borg foi arbitrariamente tida como relevante, porém ocorreu de maneira isolada em três pacientes.

\section{2) Bds e volumes pulmonares}

Após fornecimento do Bd observa-se com freqüência redução em alguns volumes pulmonares (CRF, VR), aumento em outros (CV) e elevação dos fluxos expiratórios com queda da resistência das vias aéreas.

Light et al. ${ }^{(10)}$ argumentaram que, embora outros parâmetros se elevassem mais em resposta a $\mathrm{Bd}$ do que o $\mathrm{VEF}_{1}$, este seria mais reprodutível e, portanto, mais consistente para avaliar a resposta. Guyatt et al. ${ }^{(7)}$ demonstraram por testes longitudinais que a reprodutibilidade da resposta do $\mathrm{VEF}_{1}$ a $\mathrm{Bd}$ é pobre. Além disso, a resposta média não se correlacionou com a melhora sintomática após broncodilatador.

Ramsdel e Tisi ${ }^{(41)}$ estudaram 241 pacientes com obstrução ao fluxo aéreo por espirometria e pletismografia. Uma queda significativa na resistência ao fluxo aéreo ocorreu em 129 pacientes, 46 dos quais tiveram aumento 
significativo na $\mathrm{CV}$ e queda na CRF sem mudança na relação $V_{E F} / C V F$. Os autores interpretaram esses achados como indicativos de broncodilatação refletida nos volumes pulmonares estáticos na ausência de melhora nos fluxos expiratórios. Os respondedores de volume eram mais obstruídos e mais hiperinsuflados.

Bellamy e Hutchinson ${ }^{(14)}$ avaliaram 20 pacientes com evidência radiológica de enfisema pulmonar. Após Bd houve pequeno, mas significativo, aumento no $\mathrm{VEF}_{1}$, ocorrendo elevação muito maior na $\mathrm{CV}$, que se acompanhou de redução do VR. Melhora sintomática foi relatada em 14 dos pacientes e a $\mathrm{CV}$ aumentou significativamente neste grupo $(0,55 \pm 0,39 \mathrm{~L})$, o que não ocorreu nos restantes $(0,10 \pm 0,10 \mathrm{~L} ; \mathrm{p}<0,001)$.

Connellan e Gough ${ }^{(16)}$ observaram que a melhora na tolerância ao exercício se correlacionou mais intimamente com a CV lenta do que com o $\mathrm{VEF}_{1}$. Os autores especularam, com base em dados prévios, que essa melhora poderia dever-se à redução na resistência pulmonar e CRF, resultando em vantagem mecânica aumentada para os músculos inspiratórios e redução do trabalho da respiração. Esses autores sugeriram que, quando se procura evidência de reversibilidade em pacientes com obstrução grave ao fluxo aéreo, informação útil adicional pode ser obtida simplesmente medindo a CV lenta, fluxos inspiratórios máximos, $\mathrm{Cl}$ e tolerância ao exercício antes e após Bd.

Girard e Light ${ }^{(17)}$ demonstraram que a CVF pode aumentar isoladamente após $\mathrm{Bd}$, por mais tempo expiratório ou maiores fluxos. No presente estudo, o tempo expiratório médio não variou significativamente, havendo, porém, correlação significativa entre a variação da CVF e do tempo expiratório após Bd.

Chrystyn et al. ${ }^{(15)}$ demonstraram uma relação dose-resposta evidente entre niveis de teofilina, redução da hiperinsuflação pulmonar medida pela CRF e aumento da distância percorrida em seis minutos. Esta redução na CRF refletiu-se em maior elevação da CV lenta $(630 \mathrm{ml})$ do que na CVF $(340 \mathrm{ml})$.

Gimeno et al.(11) avaliaram 51 pacientes com "enfisema" e observaram que a CPT por pletismografia não mudou e a $\mathrm{CV}$ inspiratória aumentou mais e mais freqüentemente que $\mathrm{O} \mathrm{VEF}_{1}$, porém resposta significativa do $\mathrm{VEF}_{1}$ foi considerada acima de $23 \%$ do previsto. Este limite foi obtido após medidas repetidas intra-individuais (o que mostra a pobre reprodutibilidade do $\mathrm{VEF}_{1}$ ).

\section{3) Respostas significativas a Bd}

Respostas significativas a Bd têm sido estabelecidas de diversas maneiras, porém todas se baseiam em mudanças que excedem certa variabilidade funcional da medida. As respostas usuais são expressas dividindo-se a variação pelos valores basais, pelos previstos ou como variação absoluta. Quando Bd é fornecido para indivíduos normais, a resposta situa-se abaixo de $10 \%$ do valor inicial do $\mathrm{VEF}_{1}$, sendo variável em valores absolutos e maior em homens, pelo maior tamanho corporal ${ }^{(38)}$.

Quando os resultados são expressos em percentagem de incremento em relação ao valor inicial, os aumentos serão maiores naqueles $\mathrm{com} \mathrm{VEF}_{1}$ menor e um valor único de corte é difícil de ser estabelecido, variando de $5 \%$ nos com obstrução leve a $25 \%$ naqueles com obstrução gra$v e^{(4)}$. Limite genérico de $12 \%$, baseado em um estudo pequeno, associado a variação mínima de $0,2 \mathrm{~L}$ (para contornar a dependência de expressão de resposta percentual com os valores basais), é largamente adotado(38).

$\mathrm{O}$ valor de $0,2 \mathrm{~L}$ excede o intervalo de confiança de 95\% em doentes obstrutivos considerados como um grupo ${ }^{(4)}$. Entretanto, esta variação é menor em doentes mais gravemente obstruídos e tem sido ponderado que critérios baseados na variabilidade populacional, mais do que individual, tendem a identificar apenas parte dos respondedores $^{(1)}$.

A resposta a $\mathrm{Bd}$ pode ser ainda expressa pela variação absoluta do $\mathrm{VEF}_{1}$ em relação ao valor previsto. Este índice é pouco influenciado pelo nível inicial de obstrução e corrige a resposta para o tamanho do indivíduo ${ }^{(2)}$. Valores acima de 7,5\% correlacionaram-se com incremento absoluto acima de 0,2L no $\mathrm{VEF}_{1}$ em um estudo de mais de 700 pacientes com obstrução ao fluxo aéreo(33).

Para a CVF valores acima de $350 \mathrm{ml}$ devem ser excedidos, para caracterizar resposta estatisticamente significante $e^{(3,4)}$. Inexplicavelmente, valores semelhantes aos do $\mathrm{VEF}_{1}$, de $12 \%$ do inicial e $0,2 \mathrm{~L}$, foram sugeridos pela $\operatorname{ATS}^{(38)}$ como ponto de corte também para a CVF. Quando expressa em percentagem ao valor inicial novamente, os valores diferem de acordo com o grau de obstrução(4), com um valor geral proposto de $15 \%(3)$.

Todas essas expressões de resposta a $\mathrm{Bd}$ desconsideram o significado clínico dos resultados. Respostas estatisticamente significantes não são necessariamente clinicamente relevantes.

Como visto no presente estudo e no recente trabalho

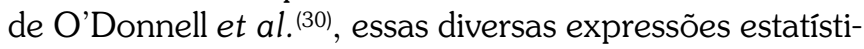
cas de resposta a Bd têm escassa relação com a melhora do desempenho no teste de exercício e devem ser abandonadas.

Respostas significativas de reversibilidade pelo $\mathrm{VEF}_{1}$, a nosso ver, deveriam ser restritas a elevações acima de $10 \%$ do previsto que não ocorrem em normais ${ }^{(38)}$, separam asma de $\operatorname{DPOC}^{(8,9)} e$, portanto, são indicativas de responsividade anormal do músculo liso das vias aéreas. Esta resposta poderia ser denominada resposta significativa de fluxo.

No presente estudo, as variações da $\mathrm{Cl}$ e da CV correlacionaram-se significativamente com os valores iniciais 
quando expressas por incremento percentual, porém esta elevação foi o melhor indicador de melhora no desempenho de exercício.

\section{4) Hiperinsuflação, volumes e Bds}

Nos anos 70 e 80 , alguns estudos ${ }^{(42,43)}$ demonstraram que, ao contrário dos indivíduos normais, pacientes com DPOC desenvolvem aprisionamento aéreo progressivo durante o esforço com CPT inalterada, portanto, com redução da CI; porém, correlação não foi feita com a dispnéia. Esta retenção aérea é denominada de hiperinsuflação por alguns autores, embora a rigor tal termo devesse ser restrito para aumento da CPT. Em estudo prévio $^{(44)}$, encontramos em 20 pacientes com DPOC avançada que a dispnéia, após caminhada, medida por escala de Borg, se correlacionava significativamente com a CRF, mas não com o VEF.

O'Donnell e Webb ${ }^{(45)}$ estudaram 23 pacientes com DPOC avançada e dez controles normais em exercício em cicloergômetro. Usando um modelo de regressão múltipla com a dispnéia como variável dependente, concluíram que o volume pulmonar endoinspirado foi o preditor mais forte. Além disso, a hiperinsuflação dinâmica explicou a variação de intensidade da dispnéia em diferentes pacientes.

Belman et al. ${ }^{(29)}$ realizaram cuidadoso estudo em 13 pacientes com DPOC antes e após broncodilatador e demonstraram que o volume endoinspirado caiu após $\mathrm{Bd} e$ este achado foi o que melhor se correlacionou com a dispnéia no exercício. $\mathrm{O} \mathrm{Bd}$ resultou ainda em aumento da reserva da pressão respiratória por elevação da $\mathrm{PI}_{\text {máx }}$. As variações nos fluxos e volumes em repouso não se correlacionaram com a variação da dispnéia no esforço.

Pellegrino e Brusasco, em 1997(12), estudaram 33 pacientes com asma e DPOC, com $\mathrm{VEF}_{1}$ acima de $40 \%$, e detectaram limitação ao fluxo expiratório por manobras de fluxo-volume lentas sobrepostas ao volume corrente. Pacientes com limitação ao fluxo aéreo tinham menor $\mathrm{VEF}_{1}$ e CVF e maior CRF. Após Bd, a limitação ao fluxo desapareceu em todos os 15 pacientes nos quais estava presente. Neste grupo a CRF e a $\mathrm{CI}$ aumentaram significativamente, o que não ocorreu nos demais.

Tantucci et al. ${ }^{(13)}$ aplicaram o método de pressão respiratória negativa na boca durante a expiração do volume corrente, para detectar limitação ao fluxo expiratório (considerado o gold standard) antes e após Bd. Em 11 de 18 pacientes com DPOC, havia limitação ao fluxo, e nestes a $\mathrm{CRF}$ caiu e a CI se elevou após Bd. Em contraste, nenhuma mudança foi observada nos sete sem limitação ao fluxo expiratório. Apenas um dos 18 pacientes teve aumento do $\mathrm{VEF}_{1}$ acima de $10 \%$ em relação ao previsto após $\mathrm{Bd}$. Estes estudos demonstraram que a limitação ao fluxo expiratório se acompanha de hiperinsuflação, que é reduzida após Bd.
Pellegrino et al., em 1998(28), estudaram 50 asmáticos e 28 portadores de DPOC e estabeleceram limites de resposta a placebo em 26 . Em 50 não respondedores pelo $\mathrm{VEF}_{1}, 13$ tiveram elevação significativa da CI.

Em 1999, O'Donnell et al. ${ }^{(30)}$ avaliaram 29 pacientes com DPOC por teste submáximo em bicicleta em carga constante e observaram que após Bd o aumento no tempo de endurance e a redução da dispnéia se correlacionaram com o aumento da $\mathrm{CI}$ medida em repouso e no exercício, mas não com a variação da CVF, PFE e $\mathrm{VEF}_{1}$. Nesse estudo, uma variação da CI acima de $10 \%$ do valor previsto foi sugerida como significativa para indicar melhor desempenho no exercício e redução da dispnéia com acurácia de 59\%.

Os resultados encontrados em nosso estudo, utilizando o teste de caminhada que tem papel longamente estabelecido na avaliação de pacientes com DPOC, são semelhantes. Pelo maior número de pacientes, pontos de corte considerando as diversas maneiras de expressão da resposta a Bd puderam ser estabelecidos mais claramente. A melhor separação foi dada pelo aumento da Cl expresso como percentagem do valor inicial, que teve acurácia de 74\%; o valor preditivo positivo foi de $91 \%$ e o negativo de $59 \%$. O encontro de teste negativo, portanto, não exclui resposta clínica, mas teste positivo assegura resposta.

A CI é determinada pelo grau de hiperinsuflação, força dos músculos inspiratórios e a extensão da carga mecânica intrínseca destes músculos. A CI também dá informação sobre a posição do volume corrente na curva de pressão-volume do SR: quanto menor a $\mathrm{Cl}$, mais próximo o VAC é da CPT e do extremo superior alinear da relação pressão-volume.

O valor da CV lenta não foi relatado no estudo de O'Donnell et al. ${ }^{(30)}$. A CPT em geral permanece inalterada após $\mathrm{Bd}^{(11-13)}$, de modo que a redução na $\mathrm{CRF}$ se reflete diretamente no aumento da CI após Bd. Em nosso estudo o VRE se elevou significativamente após Bd (em média, $0,11 \mathrm{~L}$, variando de $-0,76 \mathrm{a}+0,88 \mathrm{~L}$ ), o que se refletiu em vários casos na elevação isolada da CV. A CPT pode ser reduzida após $\mathrm{Bd}$. Em casos individuais temos observado por pletismografia, após Bd, queda do VR associada à queda da CPT e aumento da CV, com CI pouco alterada. Ramsdell e Tisi( ${ }^{(4)}$ observaram em respondedores de volume, por pletismografia, que o VR, após $\mathrm{Bd}$, caiu mais $(-853 \mathrm{ml})$ que o aumento da CV $(+465 \mathrm{ml})$, implicando redução da CPT.

No presente estudo, a CV lenta separou os respondedores dos demais pacientes e detectou respostas não verificadas pelas medidas da CI. Estes pacientes apresentaram desempenho significativamente melhor no teste de caminhada. Além disso a variação da CV se correlacionou com a variação da dispnéia, o que não ocorreu com a CI. 


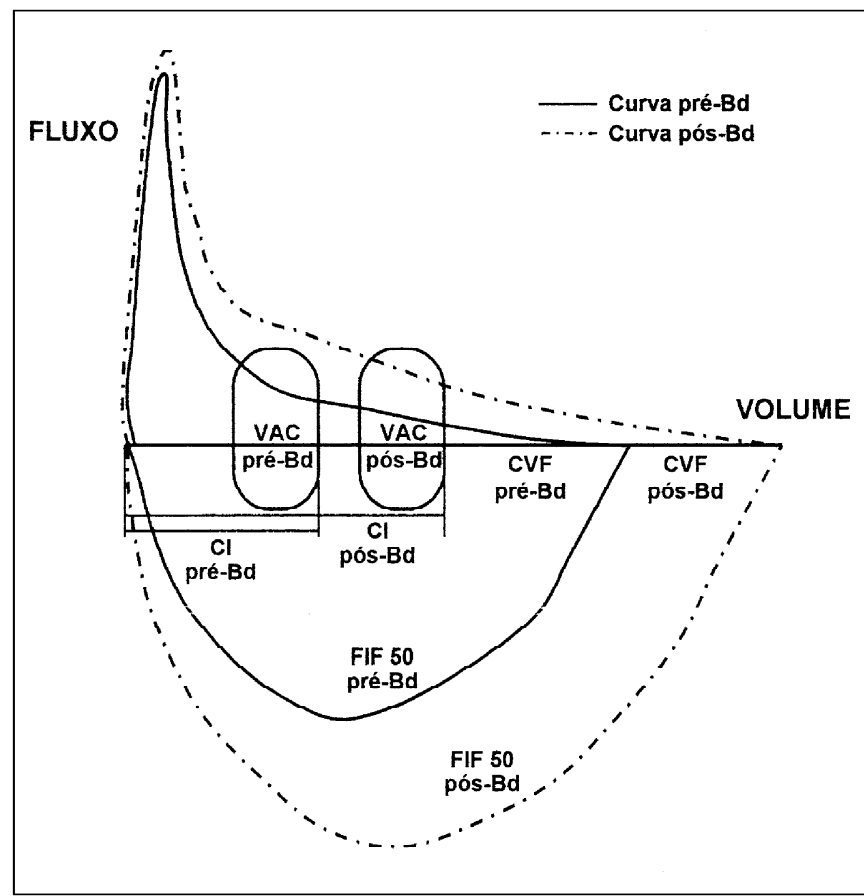

Figura 2 - Resposta a $B d$ na curva fluxo-volume com: 1) aumento da $\mathrm{Cl}$; 2) aumento da (VF e 3) aumento dos fluxos inspiratórios.

Sugerimos que ambas, a $\mathrm{Cl}$ e a CV, sejam consideradas na avaliação da resposta ao Bd. Esta resposta poderia ser denominada resposta significativa de volume, com a observação adicional de que há provável correlação com resposta clínica.

Um caso representativo de resposta isolada de volume é mostrado na Figura 2.

\section{5) Hiperinsuflação e exercício}

Em DPOC, quando os parâmetros são expressos em relação ao valor previsto, a correlação entre $\mathrm{VEF}_{1}$, dispnéia $e \mathrm{VO}_{2 \text { máx }}$ é pobre. Esta tolerância variável ao exercício é explicada por diversos fatores ${ }^{(46)}$ :

1) Diferenças na resposta ventilatória - a ventilação excessiva em DPOC decorre de espaço morto elevado, grau de hipoxemia arterial, acidose metabólica precoce, alto custo da respiração, set point alterado para o $\mathrm{CO}_{2}$ e fontes não metabólicas, como ansiedade.

2) Diferenças nos fluxos inspiratórios máximos (FIM). O FIM reflete a potência dos músculos inspiratórios e a resistência inspiratória. Em DPOC, em geral a resistência inspiratória tem importância pequena e pode ser facilmente vencida, de modo que os fatores musculares são mais importantes. No presente estudo, a elevação dos fluxos inspiratórios após $\mathrm{Bd}$ se correlacionou fortemente com a redução da hiperinsuflação que permite melhora na relação tensão-comprimento muscular. A alça inspira- tória, portanto, forneceu informações redundantes às dadas pelas medidas da CV e CI. Por sua dependência do esforço, os fluxos inspiratórios variaram acentuadamente $e$, em média, as mudanças não foram estatisticamente significantes.

3) Variações na suscetibilidade à hiperinsuflação dinâmica (HD).

A HD depende grandemente da VE de cada indivíduo e, por isso, a VE tem particular importância. A HD resulta em respiração em altos volumes pulmonares, o que exige maior esforço muscular inspiratório.

Diversos estudos enfatizaram o papel da HD como determinante-chave da dispnéia em doenças pulmonares obstrutivas $^{(13,29,30,45)}$.

A CRF em normais resulta de forças elásticas iguais e opostas entre o pulmão e a caixa torácica. Hiperinsuflação dinâmica define um aumento da CRF acima do volume de repouso do SR devido à presença de forças dinâmicas ao final da expiração: a) constantes de tempo prolongadas do sistema respiratório, por alterações da resistência das vias aéreas e complacência pulmonar; b) duração curta da expiração; c) atividade muscular inspiratória aumentada na expiração; e d) constrição da glote ${ }^{(47-49)}$.

No exercício com o aumento da ventilação esses fatores se acentuam e passa a haver elevação progressiva da $\mathrm{CRF}$, com resultante redução da $\mathrm{CI}$, já que em geral a CPT não se altera. Em normais, ao contrário, a CRF cai no exercício, por recrutamento dos músculos expiratórios na expiração. Isto também ocorre em DPOC, mas a limitação ao fluxo expiratório torna inútil esse auxílio.

No exercício, a desigualdade da distribuição da ventilação se agrava porque o aumento da freqüência respiratória reduz a complacência dinâmica das unidades pulmonares. Quando o intervalo entre respirações sucessivas é encurtado, os músculos inspiratórios devem contrair-se e vencer inicialmente a tendência para a retração do pulmão e caixa torácica (PEEP intrínseco).

$\mathrm{O}$ alívio da dispnéia pelo uso de CPAP, mas não por IPAP, demonstra a importância do PEEPI ou carga limiar(50). A carga limiar é mais importante do que o aumento de volume, mas este se torna mais significativo se há fraqueza muscular ou fadiga ${ }^{(51)}$. O que torna a carga inspiratória diferente de outros tipos de carga respiratória é que, durante a parte inicial do esforço inspiratório, a contração dos músculos inspiratórios não produz nenhuma mudança no fluxo ou volume. O mecanismo pelo qual a carga limiar produz sensação de dificuldade inspiratória pode ser grandemente explicado pelo "desacoplamento aferente" ou "dissociação neuromecânica". Por essa teoria, o cérebro "espera" um certo nível de feedback da periferia para um dado esforço; a sensação desagradável da respiração ocorre se o sinal de feedback não bate com o esperado. $\mathrm{O}$ sinal aferente pode ser uma mudança apro- 
priada no volume, fluxo, ou simplesmente comprimento muscular como resultado do esforço inspiratório ${ }^{(51,52)}$.

$\mathrm{Na}$ ausência de hiperinsuflação dinâmica a DPOC tem pouco efeito sobre os fatores relevantes ao acoplamento neuroventilatório. Estes consistem de leve alteração na capacidade geradora de pressão dos músculos inspiratórios e aumento modesto na resistência inspiratória e expiratória. Em DPOC o sistema respiratório é forçado a operar numa faixa de volume próximo à $\mathrm{CPT}$, onde o sistema é naturalmente rígido e os músculos inspiratórios são naturalmente fracos. DPOC é ímpar no sentido de que o defeito mecânico é resistivo e afeta primariamente a expiração, porém as conseqüências mecânicas são de natureza elástica e inspiratórias ${ }^{(53)}$.

\section{6) Por que não o $\mathrm{VEF}_{1}$}

Em nosso estudo o aumento da $\mathrm{CV}(\mathrm{F})$ teve relação com o melhor desempenho de exercício, o que não ocorreu com o $\mathrm{VEF}_{1}$. A falta de correlação entre aumento do $\mathrm{VEF}_{1}$ e melhora no desempenho no exercício em DPOC não é surpreendente.

O fluxo medido durante uma expiração forçada iniciada a partir da CPT, ou a curva de fluxo-volume máxima, aumenta após $\mathrm{Bd}$ menos do que o fluxo medido durante uma manobra expiratória forçada iniciada em volumes pulmonares submáximos ${ }^{(28,54)}$. Além disso, sabe-se há longo tempo que a condutância (uma manobra que não exige expiração forçada) aumenta mais que os fluxos máximos após $\mathrm{Bd}^{(55)}$.

A resposta a $\mathrm{Bd}$ não é bem refletida pelos parâmetros da curva máxima, como o $\mathrm{VEF}_{1}$ e os fluxos instantâneos num dado volume pulmonar absoluto, por diversas razões:

1) A inspiração máxima reduz temporariamente o tônus broncomotor de repouso e a retração elástica pulmonar e da caixa torácica, especialmente se a duração da pausa pós-inspiratória é elevada(56). Se a pausa pós-inspiratória é significativa, há redistribuição do ar no pulmão para unidades mais lentas, o que deve influenciar o início da expiração $\left(\mathrm{VEF}_{1}\right)$.

2) Em pacientes com obstrução ao fluxo aéreo, o maior volume pulmonar coloca os músculos expiratórios em vantagem, o que resulta em geração de pressões elevadas e compressão do gás intrapulmonar, o que distorce a verdadeira relação entre fluxos máximos e volumes ${ }^{(57)}$. Após Bd a CPT em geral não muda, o que continua a favorecer a potência muscular expiratória.

$\mathrm{O}$ aumento da força muscular inspiratória após Bd resulta não de ação direta, mas se deve à redução do volume pulmonar ${ }^{(29,58)}$.

3) Regiões que esvaziam rapidamente contribuem desproporcionalmente para as mudanças de fluxo e volume precocemente na expiração, enquanto as regiões lentas respondem pela maior parte do fluxo e volume mais tarde na expiração ${ }^{(59)}$.

4) Durante a expiração forçada de ar dos pulmões, a taxa de fluxo alcança um valor máximo, dependente do volume pulmonar, à medida que a pressão pleural é aumentada. Em tubos com paredes elásticas, o fluxo é limitado quando a velocidade do fluxo é igual à velocidade de propagação da onda de pressão em algum ponto dentro dos tubos ${ }^{(60)}$. Este ponto é chamado "ponto de estrangulamento". A velocidade do fluxo na expiração aumenta em direção às vias aéreas centrais, pela redução da área de secção transversal, ocorrendo o contrário com a velocidade da propagação da onda, que se relaciona diretamente com a área.

Um dos parâmetros mais importantes determinantes da velocidade da onda, e daí da limitação ao fluxo, é a elastância dos brônquios no ponto de estrangulamento. Após uso de Bd ocorre redução do tônus bronquial. Embora os fluxos devessem aumentar pela broncodilatação, devido à mudança na relação área-pressão dos brônquios, o ponto de estrangulamento e a propagação da onda não mudam, o que amortece a elevação dos fluxos ${ }^{(61)}$. Em doses usuais, como no presente estudo, o Bd não muda a pressão elástica pulmonar ${ }^{(62)}$, o que poderia afetar os fluxos expiratórios.

$\mathrm{O} \mathrm{VEF}_{1}$ é um parâmetro do início da expiração forçada, expressa o esvaziamento de unidades rápidas, é influenciado pela manobra de inspiração máxima precedente (que relaxa brônquios e reduz a retração elástica) e não se situa no volume pulmonar onde ocorre a limitação ao fluxo aéreo no volume corrente de exercício. Além disso, o broncodilatador amortece a propagação da onda de fluxo expiratório.

Em baixos volumes pulmonares a retração elástica é menor. Se a musculatura lisa das vias aéreas se contrai até o ponto de fechamento, isto irá ocorrer primeiro em baixos volumes pulmonares e irá refletir-se em aumento do $\mathrm{VR}$, com queda correspondente na $\mathrm{CV}$. A redução, portanto, da CV reflete o alçaponamento de ar e, assim, o estreitamento excessivo das vias aéreas ${ }^{(63)}$. Após Bd, essas anormalidades podem ser revertidas parcial ou totalmente.

\section{7) Limitações e perspectivas}

No presente estudo foram incluídos pacientes com asma e DPOC. Em asmáticos a limitação ao fluxo tem menor relevância na hiperinsuflação. Na asma a hiperinsuflação é grandemente influenciada pela atividade dos músculos inspiratórios durante a expiração ${ }^{(49)}$. Diferentes mecanismos para a hiperinsuflação poderiam mudar o perfil de resposta ao Bd.

Sugestões recentes rigorosas para o critério de reprodutibilidade nas medidas da $\mathrm{CI}$ foram feitas ${ }^{(64)}$, mas não 
foram seguidas. Medidas de $\mathrm{CI}$ na caminhada podem ser feitas com espirômetros portáteis, porém sua maior sensibilidade deve ser comprovada. Medidas de volume por pletismografia poderiam dar informações adicionais de interesse.

O reconhecimento de que as elevações da $\mathrm{CI} e$ da $\mathrm{CV}$ se correlacionam com resposta clínica tem diversas implicações. Diferentes Bds podem ser comparados. A resposta aos corticosteróides orais e inalados e outros tratamentos para DPOC devem ser reavaliados, desconsiderando-se o $\mathrm{VEF}_{1}$ como o principal desfecho ${ }^{(65,66)}$, se o que se pretende é a melhora sintomática.

Antes disso, porém, a reprodutibilidade da resposta da $\mathrm{CI}$ e CV e sua correlação com medidas de dispnéia e qualidade de vida em estudos de longo prazo devem ser melhor estabelecidas.

\section{REFERÊNCIAS}

1. Hansen JE, Casaburi R, Goldberg A. A statistical approach for assessment of bronchodilator responsiveness in pulmonary function testing. Chest 1993;104:1119-1126.

2. Harf A. How to express the reversibility of bronchial obstruction? Eur Respir J 1992;5:919-920.

3. Sourk RL, Nugent KM. Bronchodilator testing: confidence intervals derived from placebo inhalations. Am Rev Respir Dis 1983;128:153157.

4. Tweeddale PM, Alexander F, McHardy GJR. Short term variability in $\mathrm{FEV}_{1}$ and bronchodilator responsiveness in patients with obstructive ventilatory defects. Thorax 1987;42:487-490.

5. Guyatt GH, Townsend M, Pugsley SO, et al. Bronchodilators in chronic air-flow limitation. Effects on airway function, exercise capacity, and quality of life. Am Rev Respir Dis 1987;135:1069-1074.

6. Mahler DA, Matthay RA, Snyder PE, et al. Sustained-release theophylline reduces dyspnea in nonreversible obstructive airway disease. Am Rev Respir Dis 1985;131:22-25.

7. Guyatt GH, Townsend M, Nogradi S, et al. Acute response to bronchodilator. An imperfect guide for bronchodilator therapy in chronic airflow limitation. Arch Intern Med 1988;148:1949-1952.

8. Meslier N, Rocineux JL, Six P, et al. Diagnostic value of reversibility of chronic airway obstruction to separate asthma from chronic bronchitis: a statistical approach. Eur Respir J 1989;2:497-505.

9. Siafakas NM, Vermeire P, Pride P, et al. On behalf of the task force. ERS - Consensus statement: optimal assessment and management of chronic obstructive pulmonary disease (COPD). Eur Respir J 1995;8: 1398-1420.

10. Light RW, Conrad AS, George RB. Clinical significance of pulmonary function tests. The one best test for evaluating the effects of bronchodilator therapy. Chest 1977;72:512-516.

11. Gimeno F, Postma DS, van Altera R. Pletysmographic parameters in the assessment of reversibility of airways obstruction in patients with clinical emphysema. Chest 1993;104:467-470.

12. Pellegrino R, Brusasco V. Lung hyperinflation and flow limitation in chronic airway obstruction. Eur Respir J 1997; 10:543-549.

13. Tantucci C, Duguet A, Similowski T, et al. Effect of salbutamol on dynamic hyperinflation in chronic obstructive pulmonary disease patients. Eur Respir J 1998;12:799-804.

14. Bellamy D, Hutchinson DC. The effects of salbutamol on lung function in patients with pulmonary emphysema. Br J Dis Chest 1981;75:190196.
15. Chrystyn H, Mulley BA, Peake MD. Dose response relation to oral theophylline in severe chronic obstructive airways disease. BMJ 1988; 297:1506-1510.

16. Connellan SJ, Gough SE. The effects of nebulized salbutamol on lung function and exercise tolerance in patients with severe airflow obstruction. Br J Dis Chest 1982;76:135-142.

17. Girard WM, Light RW. Should the FVC be considered in evaluating response to bronchodilator? Chest 1983;84:87-89.

18. Waterhouse JC, Pritchard SM, Howard D. Hyperinflation, trapped gas and theophylline in chronic obstructive pulmonary disease. Monaldi Arch Chest Dis 1993;48:126-129.

19. McGavin CR, Artvinli M, Naoe H, et al. Dyspnoea, disability, and distance walked: comparison of estimates of exercise performance in respiratory disease. BMJ 1978;2:241-243.

20. Wijkstra PJ, Tem Vergert EM, van der Mark TW, et al. Relation of lung function, maximal inspiratory pressure, dyspnoea, and quality of life with exercise capacity in patients with chronic obstructive pulmonary disease. Thorax 1994;49:468-472.

21. Guyatt GH, Pugsley SO, Sullivan MJ, et al. Effect of encouragement on walking test performance. Thorax 1984;39:818-822.

22. Berger R, Smith D. Effect of inhaled metaproterenol on exercise performance in patients with stabel "fixed" airway obstruction. Am Rev Respir Dis 1988;138:624-629.

23. Hay JG, Stone P, Carter J, et al. Bronchodilator reversibility, exercise performance and breathlessness in stable chronic obstructive pulmonary disease. Eur Respir J 1992;5:659-664.

24. Papiris S, Galavotti V, Sturoni C. Effects of beta-agonists on breathlessness and exercise tolerance in patients with chronic obstructive pulmonary disease. Respiration 1986;49:101-108.

25. Evald T, Keittelmann S, Sindrup JH, Lange P. The effect of inhaled terbutaline on $\mathrm{FEV}_{1}, \mathrm{FVC}$, dyspnoea and walking distance in patients with chronic obstructive lung disease. Respir Med 1992;86:93-96.

26. O'Donnell DE, Bertley JC, Chau LK, Webb KA. Qualitative aspects of exertional breathlessness in chronic airflow limitation: pathophysiological mechanisms. Am J Respir Crit Care Med 1997;155:109-115.

27. O'Donnell DE, Webb KA. Breathlessness in patients with severe chronic airflow limitation. Physiologic correlation. Chest 1992;102:824-831.

28. Pellegrino R, Rodarte JR, Brusasco V. Assessing the reversibility or airway obstruction. Chest 1998;114:1607-1612.

29. Belman MJ, Botnick WC, Shin JW. Inhaled bronchodilators reduce dynamic hyperinflation during exercise in patients with chronic obstructive pulmonary disease. Am J Respir Crit Care Med 1996;153: 967-975.

30. O'Donnell DE, Lann M, Webb KA. Spirometric correlates of improvement in exercise performance after anticholinergic therapy in chronic obstructive pulmonary disease. Am J Respir Crit Care Med 1999;160: 542-549.

31. Van Schayck CP. Diagnosis of asthma and chronic obstructive pulmonary disease in general practice. Br J Gen Pract 1996;46:193-197.

32. Sociedade Brasileira de Pneumologia e Tisiologia. I Consenso Brasileiro sobre Espirometria. J Pneumol 1996;22:105-164.

33. Pereira CAC, Barreto SP, Simões JG, et al. Valores de referência para a espirometria de uma amostra da população brasileira adulta. J Pneumol 1992;18:10-22.

34. American Thoracic Society: Standardization of spirometry. 1994 update. Am J Respir Crit Care Med 1995;152:1107-1136.

35. Bates DV, Macklem PT, Christie RV. Predicted normal values for pulmonary function. In: Bates DV, Macklem PT, Christie RV, eds. Respiratory function in disease. 2nd ed. Philadelphia: WB Saunders, 1971; 93-94.

36. Bass $\mathrm{H}$. The flow volume loop: normal standards and abnormalities in chronic obstructive pulmonary disease. Chest 1973;63:171-176. 
37. Neder JA, Andreoni S, Lerario MC, Nery LE. Reference values for lung function tests. II. Maximal respiratory pressures and voluntary ventilation. Braz J Med Biol Res 1999;32:719-727.

38. American Thoracic Society: Lung function testing: selection of reference values and interpretative strategies. Am Rev Respir Dis 1991; 144:1202-1218.

39. British Thoracic Society and Associations of Respiratory Technicians and Physiologists. Guidelines for the measurement of respiratory function. Respir Med 1994;88:165-194.

40. Wolkove N, Dajczman E, Colacone A, et al. The relationship between pulmonary function and dyspnea in obstructive lung disease. Chest 1989;96:1247-1251.

41. Ramsdell JN, Tisi GM. Determination of bronchodilation in the clinical pulmonary function laboratory. Role of changes in static lung volumes. Chest 1979; 76:622-628.

42. Potter WA, Olafsson S, Hyatt RE. Ventilatory mechanics and expiratory flow limitation during exercise in patients with obstructive lung disease. J Clin Invest 1971;50:910-919.

43. Stubbing DG, Pengelly LD, Morse JLC, et al. Pulmonary mechanics during exercise in subjects with chronic airflow obstruction. J Appl Physiol 1980;49:511-515.

44. Rosa EA, Pereira CAC. Dispnéia e achados funcionais em portadores de DPOC nutridos e desnutridos. J Pneumol 1992;18:105-110.

45. O'Donnell DE, Webb KA. Exertional breathlessness in patients with chronic airflow limitation. The role of lung hyperinflation. Am Rev Respir Dis 1993;148:1351-1357.

46. Bauerle O, Chrusch CA, Younes M. Mechanisms by which COPD affects exercise tolerance. Am J Respir Crit Care Med 1998;157:5768

47. Rossi A, Polese G, Brandi G, Conti G. Intrinsic positive end-expiratory pressure (PEEPI). Intensive Care Med 1995;21:522-536.

48. Tantucci C, Ellaffi M, Duguet A, et al. Dynamic hyperinflation and flow limitation during methacholine-induced bronchoconstriction in asthma. Eur Respir J 1999;14:295-301.

49. Gorini M, Iandelli I, Misuri G, et al. Chest wall hyperinflation during acute bronchoconstriction in asthma. Am J Resp Crit Care Med 1999; 160:808-816

50. O'Donnell DE, Sanii R, Younes M. Improvement in exercise endurance in patients with chronic airflow limitation using continuous positive airway pressure. Am Rev Respir Dis 1988;138:1510-1514.

51. Yan S. Sensation of inspiratory difficulty during inspiratory threshold and hyperinflationary loadings. Effect of inspiratory strength. Am J Respir Crit Care Med 1999;160:1544-1549.
52. O'Donnell DE. Breathlessness in patients with chronic airflow limitation. Mechanisms and management. Chest 1994;106:904-912.

53. Younes M. Determinants of thoracic excursions during exercise. In: Whipp BJ, Wasserman K, eds. Exercise: pulmonary physiology and pathophysiology. New York: Marcel Dekker, 1991;52:1-66.

54. Bouhuys A, van de Woestijne KP. Mechanical consequences of airway smooth muscle relaxation. J Appl Physiol 1971;30:670-676.

55. Stamm AM, Clausen JL, Tisi GM. Effect of aerosolized isoproterenol on resting myogenic tone in normals. J Appl Physiol 1976;40:525532.

56. D'Angelo E, Prandi E, Marazzini L, Milic E, Mili J. Dependence of maximal flow-volume curve on time-course of preceding inspiration in patients with chronic obstructive pulmonary disease. Am J Respir Crit Care Med 1994;150:1581-1586.

57. Krowka MJ, Enright PL, Rodarte JR, et al. Effect of effort on measurement of forced expiratory volume in one second. Am Rev Respir Dis 1987; 136:829-833.

58. Hatipoglu U, Laghi F, Tobin MJ. Does inhaled albuterol improve diaphragmatic contractility in patients with chronic obstructive pulmonary disease? Am J Resp Crit Care Med 1999;160:1916-1921.

59. Melissinos CGI, Webster P, Tieny K, Mead J. Time dependence of maximum flow as an index of non-uniform emptying. J Apply Physiol 1979;47:1043-1050.

60. Elliot EA, Dawson SV. Test of wave-speed theory of flow limitation in elastic tubes. J Appl Physiol 1977; 43:516-522.

61. Eng J, Gomez A, Mink S. Insensitivity of maximum expiratory flow to bronchodilation in normal dogs. J Appl Physiol 1990;68:2006-2012.

62. De Troyer A, Yernault JC, Rodenstein D. Influence of beta-2 agonist aerosols on pressure-volume characteristics of the lung. Am Rev Respir Dis $1978 ; 118: 987-995$

63. Gibbons WJ, Sharma A, Lougheed D, et al. Detection of excessive bronchoconstriction in asthma. Am J Respir Crit Care Med 1996;153. 582-589.

64. Brown RA. Derivation, application, and utility of static lung volume measurements. Resp Care Clin N Am 1997;3:183-220.

65. Lacasse Y, Wong E, Guyatt GH, et al. Meta-analysis of respiratory rehabilitation in chronic obstructive pulmonary disease. Lancet 1996 348:1115-1119.

66. Van Grunsven PM, van Schayck CP, Derenne JP, et al. Long term effects of inhaled corticosteroids in chronic obstructive pulmonary disease: a meta-analysis. Thorax 1999;54:7-14. 